\title{
INTEGRATED PROJECT DELIVERY FOR INFRASTRUCTURE PROJECTS IN PERU
}

\author{
Sulyn Gomez ${ }^{1}$, Glenn Ballard ${ }^{2}$, Nader Naderpajouh ${ }^{3}$, and Santiago Ruiz ${ }^{4}$
}

\begin{abstract}
Integrated Project Delivery (IPD) implies a transformational change of the behavior and project's means and methods used by project participants. The aim is to break down the traditional silos of construction and to improve collaboration, communication, and alignment between different stakeholders of a project. As infrastructure projects are often more complex, integration is more necessary in these projects. In this paper, the authors explore introduction of the IPD concept and its strategies into infrastructure projects in Peru, and explore the contextual nuances of the adaptation of the concept and associated challenges. While some Lean construction concepts have been increasingly adopted in Peru with support of Peru's Lean Construction Institute, there is still a lack of knowledge in the market about IPD, its principles, and tools to facilitate implementation. The authors studied a company that recently aimed to change current practices through fostering colocation of stakeholders in early stages and involvement of key partners in early stages of decision-making. Evidence shows some challenges to overcome to effectively work collaboratively in a common space. This paper explores the maturity of the industry in Peru, identifies potential challenges for implementing IPD, and proposes steps to foster integration. Proposed steps include developing a sense of community and training participants in IPD related concepts, basic principles, means, and tools as well as incentivizing participants.
\end{abstract}

\section{KEYWORDS}

Integrated Project Delivery (IPD), Peru, infrastructure projects, integration, collaboration, early involvement, co-location, new market, developing countries.

1 Graduate Student, Lyles School of Civil Engineering, Purdue University, West Lafayette, IN 479072051, USA., +1 765 775-6583 (corresponding author), sgomezvi@ purdue.edu

2 Director, Project Production Systems Laboratory (P2SL), University of California at Berkeley, Berkeley, CA 94720-1712., +1 415 710-5531, gballard@berkeley.edu

3 Lecturer, School of Property, Construction, and Project Management, Royal Melbourne Institute of Technology, Melbourne, VIC 3001, +61 484 241-228, nnp@ rmit.edu.au

4 Academy Director, Graña y Montero Group, Lima15047, Peru, +51 992-789-608, sruiz@ gym.com.pe 


\section{INTRODUCTION}

During the 80's and 90's Peru suffered an economic crisis that limited investment in infrastructure projects (World Bank 2017). Consequently, the public construction sector slowed down and traditional delivery methods such as Design Bid Build (DBB) and Design Build (DB) dominated the market (Medina 2014). Today, the Peruvian economy is growing. The construction sector grew an average annual rate of $9.7 \%$ in the last decade (INEI 2016). But the construction industry has been slow to respond to changes and continued with traditional processes. This is despite the fact that the need for investing in infrastructure projects is very high, and there is a need for change of practices to respond to this need.For example, more than $25 \%$ of the Peruvian population do not have access to drinkable water. Furthermore, as shown in Figure 1, there is a need for further investment for building projects in transportation, healthcare, energy, telecommunications, and education, with up to $\$ 1.6$ billion (USD)estimated cost of meeting those needs (Bonifaz et al. 2015). As a result, the efficiency of the construction sector as the provider of infrastructure development is critical during this transition.



Figure 1: Infrastructure Gap in Peru (Bonifaz et al.2015, "A plan to get out of poverty: National Infrastructure Plan 2016-2025”, AFIN)

Poor project performance and client dissatisfaction have been attributed to different causes such as fragmentation or "the silo effect" that obstructs coordination and integration (Walker et al. 2016; Xue et al. 2005), and lack of leaders who understand and commit to new systems (Fernandez-Solis et al. 2013). Similar issues have been reported in the Peruvian construction market; issues linked to the lack of integration and misalignment of goals between stakeholders (Canales 2014).

Integrated Project Delivery (IPD) has emerged as an alternative approach for delivering value to clients and improving overall project performance by aligning stakeholders interest and objectives (Mesa et al. 2016; Kim et al. 2016). Instances of IPD projects transitioning from an individual mindset to a collaborative approach have been documented by practitioners and academic researchers (Walker et al. 2016; Cohen 2010; Forero et al. 2015). Although IPD has primarily been applied to the design and construction phase of projects, an integrated project is shaped and delivered with an eye 
to the entire lifecycle of the constructed asset (Cohen 2010). Consequently, IPD is a good fit for infrastructure projects delivered in the form of private-public partnership (PPP) due their high complexity. One of the first practices that the company under study is fostering to make projects more integrated is co-locating major partners early in the project to work together. Early contractor involvement has been a feature of more advanced practice for some time, but adding co-location is intended to increase the benefits of that involvement, however, some challenges are observed in the process. The authors summarized a list of principles and tools (Fig. 2) that facilitate IPD implementation and present a detailed analysis of those which are being used in the Peruvian construction industry.
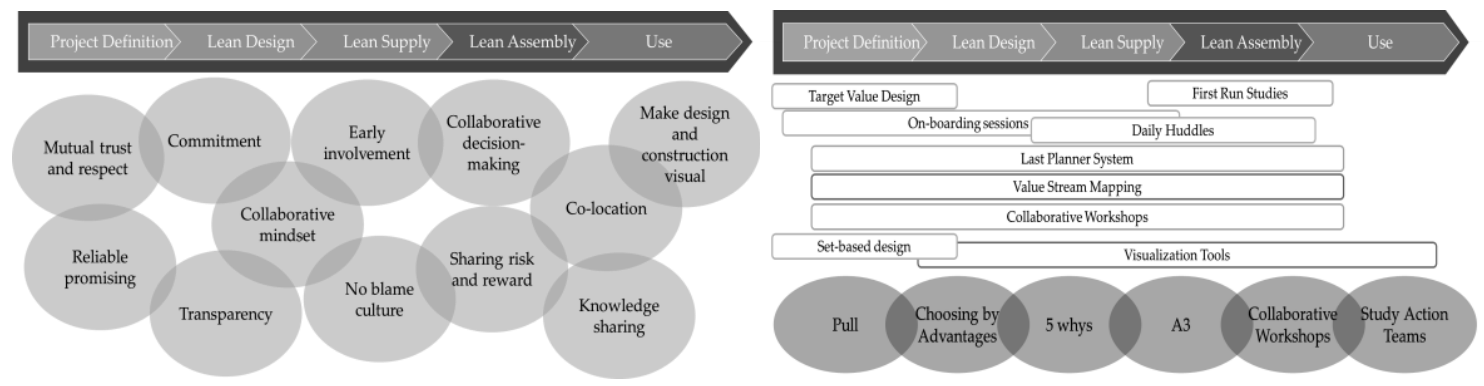

Figure 2: Principles in an IPD project and tools that facilitate IPD implementation

\section{RESEARCH DESIGN}

A case study is conducted to explore the why's and how's of the contemporary phenomenon of integration in a new market in its real-world context (Rowley 2002; Yin 2009). As IPD is relatively new in Latin America's construction industry, the case study method allows us to analyze and better understand its applicability in the Peruvian culture. The case is focused on a Peruvian holding company composed of firms with development, engineering, construction, and operations/maintenance missions and competencies. While the company delivers the PPP project largely by itself, the challenge is to coordinate and integrate the involved firms to act for the good of the whole, and not try to suboptimize each of their parts. Main obstacles to integration include:(1) incompatibility of the current management system for integration, (2) lack of procedures that instigate integration, and (3) need for alignment mechanisms (KPMG 2013). Consequently, integration need to be fostered through the structure of the organization to improve project performance and quality of work environment.

As part of the case study, the authors conducted an extensive survey of Peruvian professionals involved in the delivery of the studied infrastructure division. The goal was to gauge the stakeholders' perception and expectations for being involved in a collaborative delivery model and currently applied practices. Through the survey outcomes and field observation, the cultural context and current practices were investigated and further steps for improvement were developed. These further steps are presented in the conclusions section of this paper. Thirty people (30) from the case study were invited to participate in the survey; 26 of them responded. The number of respondents by position were: project managers (16), designers (2), construction 
managers (2), field engineers (3) and technical office engineers (3). Examples of infrastructure projects in Peru that the participants have been involved in include: Linea 1 metro of Lima (PPP), Linea 1 expansion project (PPP), Linea 1 operations concession (PPP), Linea 1 second expansion (PPP), Cayetano Heredia hospital (PPP), Quellaveco mining project, and Cerro del Aguila hydroelectric project.

\section{RESEARCH FINDINGS}

\section{LEVEL OF COMPLEXITY}

Integration between parties is shaped differently as project's complexity and uncertainty increases (Mitropoulos and Tatum 2000). The survey included a question with the Likert scale rating between 1 to 5measure the respondents' perception of project complexity. This question was included to confirm the assumption that infrastructure projects are highly complex. It seems likely that high complexity can either motivate and encourage people to perform better or demotivate them should they think that the tasks are far from their capabilities. Furthermore, the perception of the level of complexity might trigger the change in behaviors labeled as "This is how I've always done it" (Fernandez-Solis et al. 2013).As shown in Figure 3, the participants reported that the projects (all in the infrastructure division) have a medium to high complexity. It has been argued that increased complexity of projects requires collaborative and creative behaviors in order to be successful (Ballard et al. 2011). Further, the IPD delivery system has demonstrated its capabilities in dealing with complex projects (Cohen 2010; Mesa et al. 2016; and Ballard et al. 2011). However, there are questions how to promote the desired behaviors.

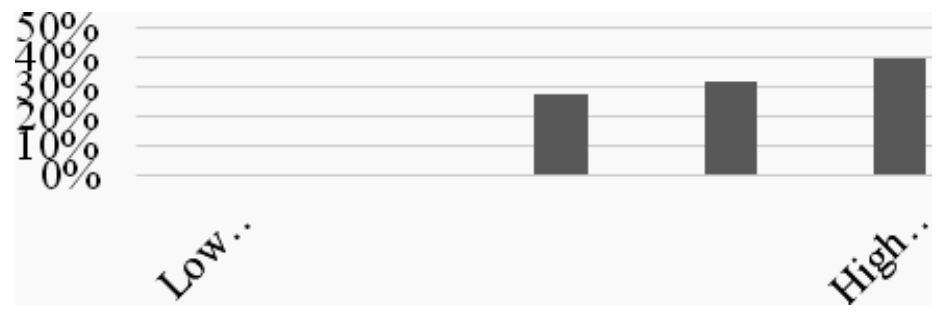

Figure 3: Level of complexity

\section{LEAN, BIM, AND IPD LEVEL OF KNOWLEDGE}

The study also analyzed the level of familiarity of the participants with Lean, BIM, and IPD. The results for each category is shown in Table 1. As the studied company has been implementing Lean Construction for some years, most of the participants (93\%) were familiar with Lean and used it to some extent. On the other hand, around two thirds of the participants were familiar with the concept of IPD or have only heard about it. Therefore, to encourage successful take up of the concept in the new market, training would play a crucial role. 
Table 1: Knowledge of Lean, BIM and IPD within the participants

\begin{tabular}{cccc}
\hline Answer & LEAN & BIM & IPD \\
\hline Yes & $93.3 \%$ & $73.3 \%$ & $40.0 \%$ \\
A little bit & $6.7 \%$ & $20.0 \%$ & $26.7 \%$ \\
No & $0.0 \%$ & $6.7 \%$ & $33.3 \%$ \\
\hline
\end{tabular}

\section{TOOLS FOR FACILITATING IPD IMPLEMENTATION}

Projects using IPD employ multiple tools (Fig. 2)to facilitate implementation of IPD even when the level of awareness about these tools as part of IPD is very low. The results shown in Figure 4 support what the researchers have observed during site visits in the company. Generally, there is a lack of knowledge in different projects about tools available for use in the projects. Even though most of the participants (89\%) are aware of the Last Planner System, there seems to be considerable opportunity to start using the big room effectively, while there is also a need to establish an on-boarding process for the whole team. Studies suggests also that tool such as co-location, on-boarding, A3, and CBA can potentially impact a successful implementation of IPD in projects (Cheng 2016). Therefore, the efforts of the company in fostering co-location might need an adjustment to make it more effective.

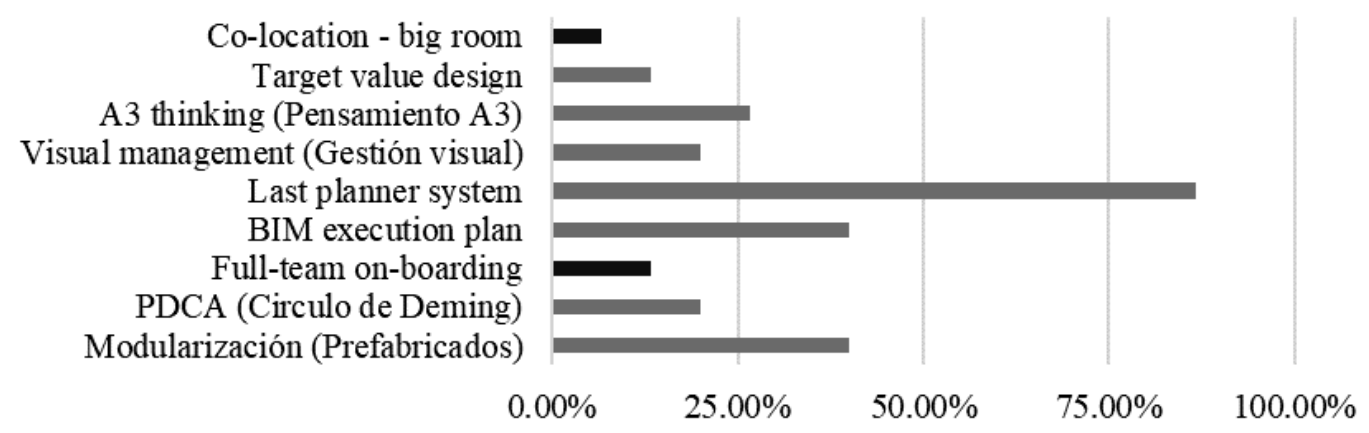

Figure 4: Tools being used by survey respondents

\section{CASE STUDY: CO-LOCATING AND COLLABORATIVE DECISION-MAKING}

Fully "93.3\%" of survey respondents considered the early involvement of project stakeholders very useful. As part of the case study in the company, the researchers focused on "Project A", the first attempt to apply IPD in the organization. Project A involved major stakeholders early in the project as shown in Fig. 5. Specifically, the engineering and construction companies engaged the operations and maintenance company to work with them early on. 


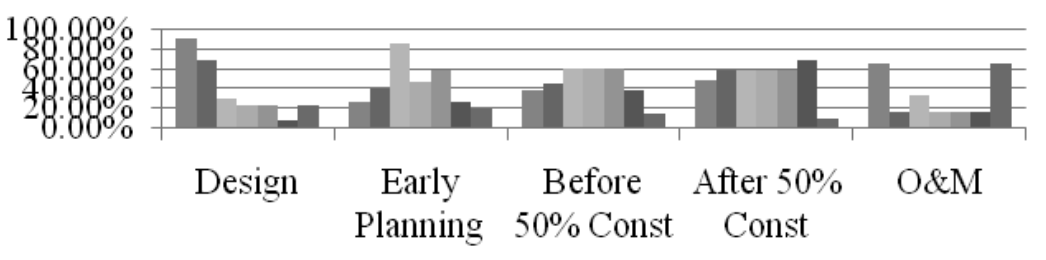

Figure 5: Co-location of participants for project A

\author{
Owner \\ - Designer \\ General Contractor \\ MEP Contractor \\ - Structural Contractor \\ - Finishing Contractor \\ - O\&M Contractor
}

As developing highly effective meetings is fundamental when co-locating the project teams, the researchers asked the participants how they would improve the team meetings. The responses varied from "attending meetings with a mindset of looking for solutions instead of imposing ideas on others", "attending on time and having all the decisionmakers coming to the meeting", "involving people actively and keeping a weekly routine", "having short meetings previous to the general one and making sure commitments are completed in advanced", "establishing commitments with the project team", "improving accomplishment of activities committed in the previous week and removal of constraints". The common theme among the observed concerns of the participants was about bringing people together and keeping track of their task compliance. IPD works when individuals are in the social exchange framework to make and keep commitments (Mossman et al. 2011).The process of improving how meetings are held may also help to transform the culture to one of increased trust, collaboration, and shared learning.

Co-locating parties impact positively the decision-making process. Involvement of different parties in making decisions was also explored in the study (Fig.6).Results show that there was more involvement in making decisions in early phases than later. Team members' level of involvement varied in decisions concerning project scope, cost, schedule, change orders and interferences. Therefore, when compared to traditional approaches, there seems to be an improved in bringing stakeholders earlier to improve project delivery.
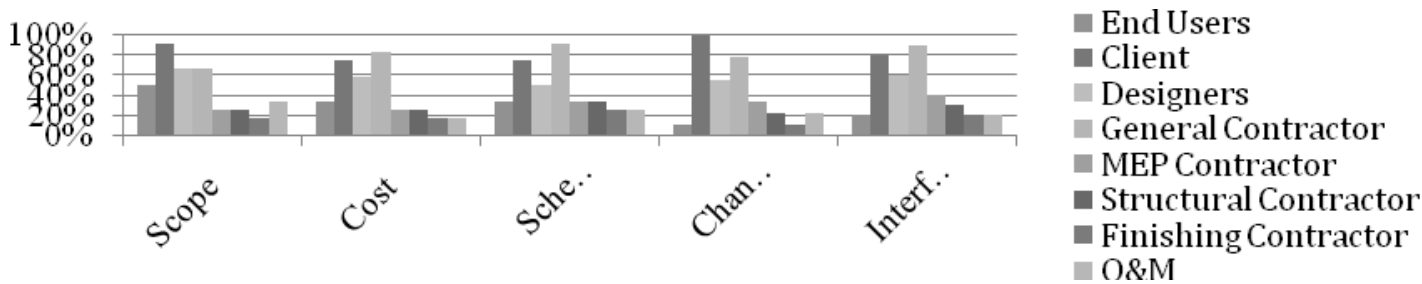

Figure 6: Parties involvement in decision-making process

In Figure 7, the main drivers that the participants consider when selecting key partners for their project were explored. As compared to traditional mindset in construction where the main driver tends to be only cost, the new approach is pushing companies to consider factors such as technical proposal, expertise, and design. When dealing with complex projects such as Project A, a diverse set of people need to be involved in the team that can potentially create a more creative solution to deal with the high level of project 
complexity. In Project $A$, the team had $\$ 9$ million at risk, therefore converting risks into opportunities was considered necessary for all parties.

For a more accurate and precise selection of key partners, tools shown in Figure 4such as CBA and A3can be applied that will help the team to take more informed decisions based on the advantages to the project. Moreover, on-boarding processes are needed when bringing people to get involved in early stages of the project (Seed 2015), which allows participants to comprehend the process and its effectiveness while getting used to new tools.

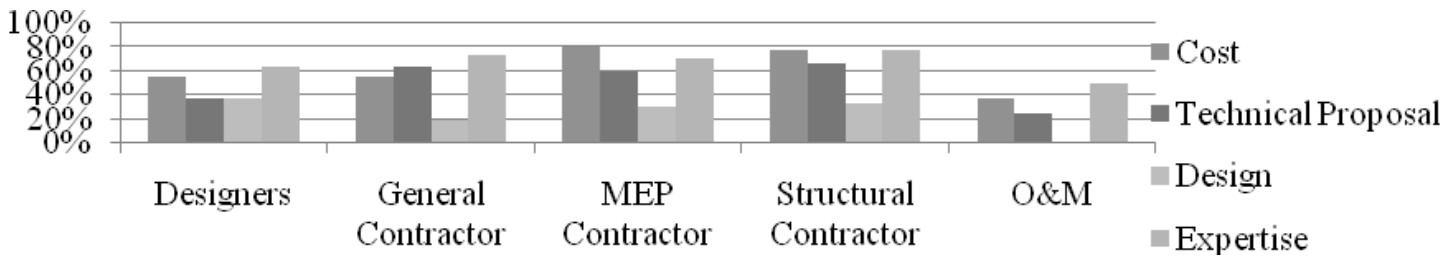

Figure 7: Factor considered for choosing partners

\section{ESSENTIAL FACTORS FOR IMPLEMENTING IPD}

The author aimed to understand the participants' perception about key principles or factors to achieve an integrated project and delivering a successful project. They were given a multiple-choice question, with the option to include additional choices. A summary of the responses is presented in Fig.8. Surprisingly, "respect" was the lowest factor rated by participants which suggested the need to change the current mindset regarding core values. However, this answer could have also be received due to a misunderstanding by the respondents since one of them later stated that respect was already implicit in the way they behave and it was not seen as a separate principle. IPD necessitates cultural change through

a more collaborative mindset as it requires new ways of behaving and thinking (Ballard 2008; Pishdad-Bozogi 2016). In a scenario where survival is more important than working conditions, there is a need to go back to basics and keep in mind that Lean requires respect.

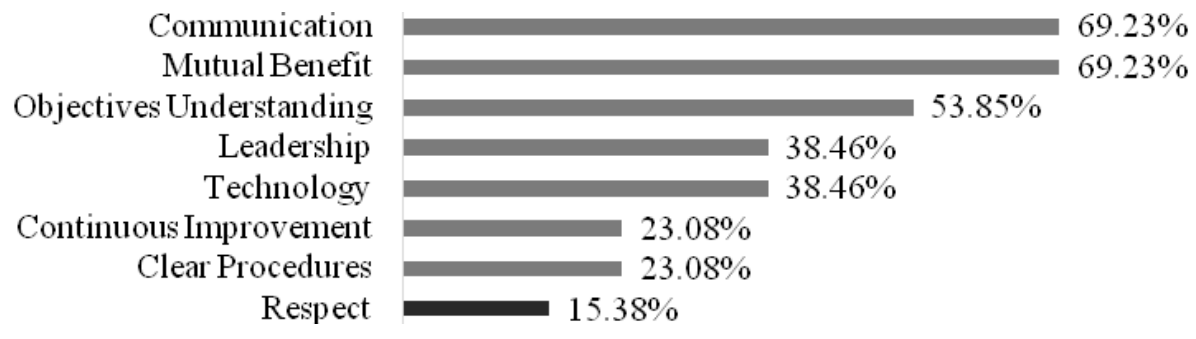

Figure 8: Essential factors for implementing IPD successfully

\section{WILLINGNESS TO CHANGE}

The vision for changing to a more collaborative model was strengthened by the company's willingness to deliver the highest engineering value to their clients and 
increase the level of service for their infrastructure proposals. The business developer company of the group is leading the initiative as an internal client for the other companies of design, construction and operations. The authors aimed to understand team member's expectations for change and where they had identified potential areas for improvement.

To identify participants' motivation to change their current practices, the authors asked them to comment on how they would describe a successful day in their project. Responses varied from "Improve communication and daily commitments accomplishment", "constraints removal and sharing progress with the team and getting the work done", "keep a constant work flow", "zero accidents, zero rework, high productivity", "implementing innovative processes". Common patterns among these responses suggest that stakeholders' perspective about a successful day in their projects differ from each other which means that there is a misalignment of goals and therefore priorities might be completely different. While some people concern lies on improving communication, other members' focus is more productivity oriented such as avoiding accidents and rework.

The researchers also asked the participants "If you could change something in the project, what would it be?". Responses to this question varied from "Increase client involvement and accurate communication with client", "schedule activities with the different disciplines", "define clear rules when working with the different companies in the group", "improve contract clauses in order to make it more collaborative instead of aggressive", "improve constraints analysis and planning process", "commitment compliance, improve daily planning and share it with the team", "share project goals more often", "effective communication", "involve all stakeholder earlier in the project to elaborate an integrated planning and execution program", "planning should consider all different variables that might impact it". Common patterns among these responses suggest:

- Project teams have realized the need to get the owner involved in the development and execution of the projects. A key characteristic from IPD projects is the level of commitment that owner ensures in the process. For infrastructure projects in the way of PPP in Peru where the client is the government, there is the figure of an internal client (business developer) who can play the role of the final client. However, there is a need to empower this player for facilitating decision-making.

- By requesting rules clarification, there is a suggestion to improve the company's contracts and guidelines. Though having an IPD type of contract with the government might be a difficult if not impossible fact under the current legislation, the organization can still work on an internal agreement between their companies that can facilitate key IPD strategies implementation such as sharing risk and reward, and clarify roles and responsibilities within teams.

- Even though the company has been implementing Lean tools and especially Last Planner System through the organization, basic things such as constraint analysis, still need to be reinforced by training and encouraging collaboration between the different disciplines to avoid interreferences and rework. 


\section{DISCUSSION AND CONCLUSIONS}

The authors discussed points such as the understanding of the definition of "respect" by participants. It may be hypothesized that the culture of survival that still prevails in most companies might be reason for low rate of this metric in the study. In such case, it would be important to know how can we build respect in an industry that has been seriously damaged with corruption nowadays? Also, is there any relation between open communication and effective communication? While the level of communication might be acceptable, it may not be through the most effective channels. In the case study it was observed that despite the implementation of the practice of co-locating parties, communication did not flow as expected. We hypothesized that other practices such as visual management may need to be implemented to facilitate communication of colocated parties. It is also interesting to explore how trust will influence predisposition of people to share risks and rewards. Some steps for improvement have been suggested (Fig. 9) such as reinforcing the use of LPS to improve reliability and foster training programs in TVD to set and steer to targets in integrated projects.

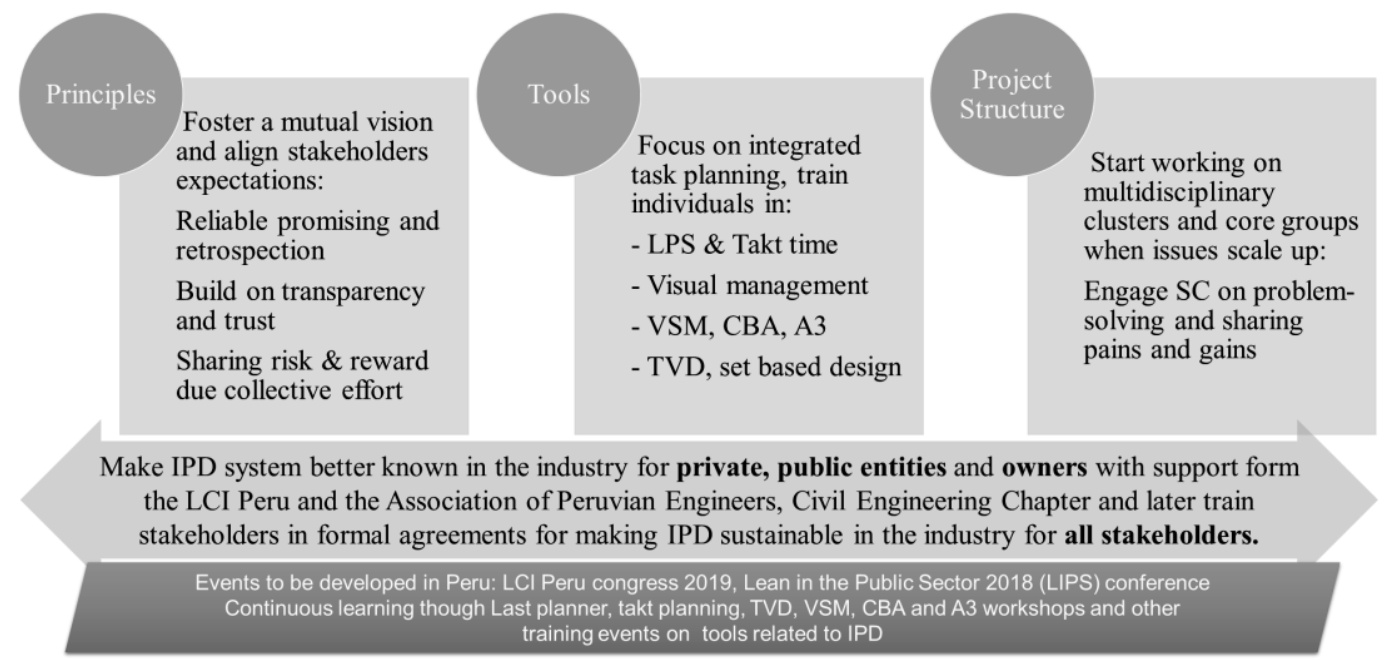

Figure 9: Steps for improve IPD implementation in Peru

The collaborative approach aims to meet higher service levels and improve the current process for design, construction and operation of infrastructure projects. The study only included data from one company, which is the most experienced using Lean construction. Results of this study should not be generalized, rather the findings should be used to give an overview of IPD implementation in Peru. Even tough Peru faces issues that seem to be characteristics of every place where construction is done, the different cultural settings (motivation and barriers) from country to country might require different triggers for generating collaboration and what might vary in fact is the process that will be adopted to achieve the required level of integration. The research reported in this paper explored practical nuances of IPD and key points where changes are required are summarized below: 
- Complexity as a catalyst for moving to a collaborative approach: Even though a complex project may be more unpredictable and challenging, the high level of complexity of infrastructure projects may act as a catalyst to motivate a disruption in the dynamics of project teams towards more integration.

- Lack of knowledge in regard to IPD: Dealing with the lack of knowledge regarding IPD in the Peruvian sector requires more effort to train people to better understand the concept. The training process might increase awareness of potential use of tools that facilitate IPD. Even though the participants have started collaborating early in the construction process, the multidisciplinary teams can improve the integration practices by using tools such as A3, CBA, and PDCA.

- Co-locating team members from early stages: To improve efficiency of the colocation process (big room meetings), several rules exist that can be applied to the project team such as safe zone sense, importance of the opinion of every individual, and sense of equality in the status of the individuals.

- Dealing with a culture of survival, fostering "respect": The current state where a culture of survival prevails need to be transformed by adopting a collaborative approach which will change people's mindset with a focus on respect. Accountability, trust, and respect need to be strengthened with the support of making/keeping real commitments. However, trust and respect might imply different behavioural patterns in different institutional contexts. The company under study has started a leadership program to reinforce the concept of respect as a recognized sovereign right of people to think differently.

- Work on basics: According to the perception of participants, there is a need to improve reliability in the projects. For this purpose, the planning process need to be improved. It was observed that people are still following the "push" pattern of behaviour in some projects and not removing constraints properly. The authors recommend the initiation of redesigning the production system considering different requirement of the involved parties and their disciplines.

- Commercial terms: Participants suggested that there is a need to improve the regulative frameworks that support IPD in the construction industry. Internal agreements in the form of addendums can be used to promote collaboration.

\section{REFERENCES}

Cohen, J., 2010. "Integrated Project Delivery: Case Studies." American Instituteof Architects (AIA) - AIA California Council, Sacramento, CA.

Ballard, G. (2008). "The Lean Project Delivery System: An Update."Lean Construction Journal.

Ballard, G., Kim, Y., Azari, R., and Cho, S. (2011). Starting from Scratch: A New Project Delivery Paradigm., Research Report 271, Construction Industry Institute, Austin, Tx. Bonifaz, J., Urrunaga, R., Aguirre, J., Urquizo, C., Carranza, L., Laguna, R., Orozco, A., (2015) "A plan to get out of poverty: National Infrastructure Plan 2025" AFIN institute. 
Canales, J., (2014). "Effects of innovative contracts into the supply chain performance in the Peruvian construction industry."Master Diss. Politecnico di Milano, Italy.

Cheng, R. (2016). Motivation and Means: How and Why IPD and Lean Lead to Success. Lean Construction Institute (www.leanconstruction.org)

Fernandez-Solis, J., Porwal, V., Lavy, S., Shafaat, A., Rybkowski, Z., Son, K. (2013). "Survey of Motivations, Benefits, and Implementation Challenges of Last Planner System Users".ASCE, J. Constr. Eng. Manage., CO.1943-7862.0000606, 354-360.

Forero, S., Cardenas, S., Vargas, G and Garcia, C. (2015). "A deeper look into the perception and disposition to integrated project delivery (IPD) in Colombia." Proc. 23rd Ann. Conf. of the IGLC group. Perth, Australia, pp. 297-306.

INEI. (2016). Peru: Statistical Summary 2016.National Institute of Statistics and Informatics, Lima, Peru.

Kim, Y-W., Rezqallah, K., Lee, W and Angeley, J. (2016). "Integrated project delivery in public projects: Limitations and opportunity." Prc $24^{\text {th }}$ Conf. IGLC group. Boston, USA

Medina, A., (2014). "Learning Through Failure: TheChallenge of Lean Project Delivery from the Contractor's Perspective inPeru." Proc. $22^{\text {nd }}$ Conf. IGLC group. Oslo, Norway

Mesa, H., Molenaar, K., and Alarcón, L., (2016)."Exploring performance of the integrated project delivery process on complex building projects. "Int'1 Jr of Project Management.

Mitropoulos, P., and Taum, C. B. (2000). "Management-driven integration." Journal of Management in Engineering, 16(1), 48-58

Mossman, A., Ballard, G. and Pasquire, C. (2011). The growing case for Lean construction. Construction Research and Innovation V.2(4), pp.30-34.

Pishdad-Bozorgi, P., and Beliveau, Y. J. (2016). "A Schema of Trust Building Attributes and Their Corresponding Integrated Project Delivery Traits." Intl. Journal of Const. Edu. and Research, Georgia Institute of Technology, Atlanta, USA, 12(2), 142-160.

Rowley, J. (2002). "Using Case Studies in Research", Manage. Research News, 25, 16-27.

Seed, W. R. (Ed.). (2015). Transforming design and construction: A framework for change. Lean Construction Institute.

Walker, D. H. T., Davis, P. R., and Stevenson, A. (2017). Coping with uncertainty and ambiguity through team collaboration in infrastructure projects. International Journal of Project Management, 35(2), 180-190.

World Bank Group.(2017). Peru Systemic Country Diagnostic (SCD), report 112694-PE

Xue, X., Li, X., Shen, Q., Wang, Y., 2005. An agent-based framework for supply chain coordination in construction. Autom. Constr. 14 (3), 413-430.

Yin, R. K., 2009. Case Study Research: Design and Methods. Thousand Oaks, CA: Sage. 